\title{
Chiroptical interactions between twisted light and chiral media
}

Kayn A. Forbes, David L. Andrews

Kayn A. Forbes, David L. Andrews, "Chiroptical interactions between twisted light and chiral media," Proc. SPIE 10549, Complex Light and Optical Forces XII, 1054915 (22 February 2018); doi: 10.1117/12.2290134

SPIE. Event: SPIE OPTO, 2018, San Francisco, California, United States 


\title{
Chiroptical interactions between twisted light and chiral media
}

\author{
Kayn A. Forbes and David L. Andrews* \\ School of Chemistry, University of East Anglia, Norwich NR4 7TJ, United Kingdom
}

\begin{abstract}
The extent to which structured light might conceivably resolve the handedness of chiral matter is a topic of resurgent interest, and it is one that has been a challenge since the earliest days of optical vortex studies. It has not even been certain whether or not the orbital angular momentum of light could interact in such a way - though it has been established that electric quadrupole interactions enable twisted light to engage with local electronic transitions. Crucially, certain recent experiments have provided tantalizing evidence to support the existence of a chiral effect that is sensitive to handedness, against initial expectations. By detailed electrodynamic calculation, a new study has now fully identified the mechanism, and also provided an in-depth analysis of the role of electric quadrupole transition moments as they engage with the phase gradient of beams with a twisted wavefront. Focusing on single photon absorption, it emerges that the orbital angular momentum associated with the vorticity of a structured beam can indeed be exhibited in chiral effects, provided the material itself is not only chiral, but also has some structural order - which essentially limits the effect to chiral solids, poled liquid crystals, and oriented arrays of chiral nanoparticles. Circular polarization is still required, and the extent of circular dichroism proves to vary around the beam, being locally determined by the absorber orientation with reference to the beam axis. In agreement with earlier studies, and consistent with symmetry principles, the new analysis verifies that any dependence on wavefront vorticity vanishes in a freely mobile fluid. The reformulation of theory now paves the way for an extension to other kinds of chiroptical phenomena in orientationally ordered systems.
\end{abstract}

Keywords: Chirality, twisted light, optical vortex, nanoparticle, liquid crystal, photon interactions, optical activity, orbital angular momentum

\section{INTRODUCTION}

The optical orbital angular momentum of light is now a well-established and flourishing research field within optics. ${ }^{1-3}$ An inference that light could possesses an orbital angular momentum (OAM), alongside the more familiar and intrinsic spin angular momentum (SAM), was known during the early development of quantum theory, ${ }^{4}$ being understood to manifest in multipolar transitions. However, it is now widely agreed that the contemporary prominence of this field has its origins in the pioneering work of Allen, et. al. where it was shown that is should be possible for laser light to be prepared in the laboratory with a well-defined orbital angular momentum. ${ }^{5}$ In contrast to the SAM of light, which has helicity eigenvalues restricted to $\pm \hbar$ per photon and which is manifest through circularly polarized photon states, the OAM associated with the different forms of structured light has no such bounds on dimensionality, ${ }^{6}$ each photon being able to possesses values of $\pm \ell \hbar$, where $\ell$ is any integer. The quantity $\ell$ represents the topological charge that characterizes the wavefront structure, the higher the value the more the beam 'twists' within a wavelength. 
Chiroptical interactions involve the interplay of the chirality of the molecular and radiation components of the total lightmatter system to produce energy shifts and optical rates that are sensitive to changes in handedness. ${ }^{7}$ For the material component of the total radiation-matter system, the molecules (or unit cell in the case of a solid) are said to be chiral if they have no improper axis of rotation i.e. they are non-super imposable mirror images of one another, and are designated either right- or left-handed. The chirality of light can be manifest in two ways: the sense of turning direction of the electromagnetic field vectors in a circularly polarized beam, being either clockwise (right-handed) or anticlockwise (left-handed); or the sense of direction that the helical wavefront is twisting in a beam possessing orbital angular momentum: beams possessing $\ell>0$ are left-handed, whilst $\ell<0$ are right-handed.

To date, practically all chiroptical phenomena are known to involve the engagement of molecular chirality with light's handedness through circularly polarized photons. Classic examples being circular dichroism ${ }^{8,9}$ and differential scattering, ${ }^{10}$ with more contemporary studies involving the optical nanomanipulation techniques known as optical trapping and optical binding. ${ }^{11,12}$ The notion of whether the handedness of a twisted beam could play a determining role in a chiroptical process has been an issue much-studied previously. ${ }^{13-16}$ Early studies determined that for molecules interacting with structured light, the sign of the topological charge of a Laguerre-Gaussian beam (LG beam) produces no discriminatory effects. This theoretical result was subsequently further reinforced by experimental studies. ${ }^{17,18}$ The theoretical work, however, centered on the issue of whether the electric and magnetic dipole moments (written as E1 and M1 in shorthand notation) could engage with the handedness of the vortex beam. At this level of representation, it can be asserted that these forms of coupling indeed produce no such effect, either for molecular systems which possess either a degree of order, or conversely those that are completely randomly oriented. However, by inclusion of electric quadrupole moments (E2) in the analysis presented here, it can now be shown that the engagement of E2 moments with structured light secures a result which does indeed highlight the chirally sensitive role of the handedness of the vortex beam.

\section{TWISTED BEAMS INTERACTING WITH CHIRAL MEDIA}

To elucidate the mechanism of which the handedness of the vortex beam plays a role in chiroptical interactions, we will study the most basic of optical interactions, namely single-photon absorption. In the following work, we employ the Power- Zienau -Wooley (PZW) Hamiltonian to describe the photon-molecule interaction. Ignoring a self-energy correction term, which plays no part in radiative processes, the PZW Hamiltonian takes the form of a sum of Hamiltonians for the molecule, radiation, and the mutual interaction between the two:

$$
H^{\text {mult }}=H_{\text {mol }}^{\text {mult }}+H_{\text {rad }}^{\text {mult }}+H_{\text {int }}^{\text {mult }} .
$$

The PZW Hamiltonian has the distinct advantage of describing photon-molecule interactions due to the interaction Hamiltonian term being expressed in multipolar form, i.e.

$$
H_{\mathrm{int}}^{\mathrm{mult}}=\sum_{\xi}\left[-\varepsilon_{0}^{-1} \boldsymbol{\mu}(\xi) \cdot \boldsymbol{d}^{\perp}\left(\boldsymbol{R}_{\xi}\right)-\varepsilon_{0}^{-1} Q_{i j}(\xi) \nabla_{j} d_{i}^{\perp}\left(\boldsymbol{R}_{\xi}\right)-\ldots-\boldsymbol{m}(\xi) \cdot \boldsymbol{b}\left(\boldsymbol{R}_{\xi}\right)-\ldots\right]
$$

where the term involving $\mu(\xi)$ is the electric dipole coupling E1 for a molecule $\xi$; the second term involving the electric quadrupole moment $Q_{i j}(\xi)$ is the E2 coupling; and the final term is the magnetic dipole $\boldsymbol{m}(\xi)$ (M1) interaction. 
Retention of only the E1 term is the well-known electric-dipole approximation. To the level of multipole moment expansion that is included above, there is also an additional lowest-order diamagnetic coupling term. We have neglected this diamagnetic coupling term in the following analysis as it plays no role in single-photon absorption due to it possessing a quadratic dependence on the magnetic field, and therefore its inclusion is only necessary in optical processes involving two or more photon creation/annihilation events. ${ }^{19}$

To take account of the properties of structured light, the well-known vacuum mode expansions for the transverse electric displacement field $\boldsymbol{d}^{\perp}$ and the magnetic field $\boldsymbol{b}$ need to be duly modified. We confine our analysis to the paraxial approximation, whereby it is legitimate to assume that laser light propagation consists of wave vectors which have negligible inclination to the optical axis. As such, one of the most widely utilized solutions to the paraxial wave equation are the Laguerre-Gaussian modes - these are the most common modes for generating and describing laser beams with an orbital angular momentum. The electric displacement and magnetic field expansions for LG beams in the paraxial approximation emerge as functions of the cylindrical $\operatorname{coordinates}^{20}$ : the off-axis radial distance $r$, axial position $z$, and azimuthal angle $\phi$

$$
\boldsymbol{d}^{\perp}(\boldsymbol{r})=i \sum_{\boldsymbol{k}, \eta, \ell, p}\left(\frac{\hbar c k \varepsilon_{0}}{2 V}\right)^{1 / 2}\left[\boldsymbol{e}_{\ell, p}{ }^{(\eta)}(\boldsymbol{k}) a_{\ell, p}{ }^{(\eta)}(\boldsymbol{k}) f_{\ell, p}(r) \mathrm{e}^{(i k z+i \ell \phi)}-\overline{\boldsymbol{e}}_{\ell, p}{ }^{(\eta)}(\boldsymbol{k}) a_{\ell, p}{ }^{\dagger(\eta)}(\boldsymbol{k}) \bar{f}_{\ell, p}(r) \mathrm{e}^{-(i k z+i \ell \phi)}\right],
$$

and

$$
\boldsymbol{b}(\boldsymbol{r})=i \sum_{\boldsymbol{k}, \eta, \ell, p}\left(\frac{\hbar k}{2 \varepsilon_{0} c V}\right)^{1 / 2}\left[\boldsymbol{b}_{\ell, p}{ }^{(\eta)}(\boldsymbol{k}) a_{\ell, p}{ }^{(\eta)}(\boldsymbol{k}) f_{\ell, p}(r) \mathrm{e}^{(i k z+i \ell \phi)}-\overline{\boldsymbol{b}}_{\ell, p}{ }^{(\eta)}(\boldsymbol{k}) a_{\ell, p}^{\dagger(\eta)}(\boldsymbol{k}) \bar{f}_{\ell, p}(r) \mathrm{e}^{-(i k z+i \ell \phi)}\right],
$$

where $f_{\ell, p}(r)$ is the radial distribution function for a paraxial beam of waist $w_{0}$

$$
f_{\ell, p}(r)=\frac{C_{p}^{|\ell|}}{w_{0}}\left[\frac{\sqrt{2} r}{w_{0}}\right]^{|\ell|} \mathrm{e}^{\left(-r^{2} / w_{0}^{2}\right)} L_{p}^{|\ell|}\left(\frac{2 r^{2}}{w_{0}^{2}}\right)
$$

$a_{\ell, p}{ }^{(\eta)}(\boldsymbol{k})$ and $a_{\ell, p}^{\dagger(\eta)}(\boldsymbol{k})$ are the annihilation and creation operators for a photon of mode $(\boldsymbol{k}, \eta, \ell, p)$.

Restricting consideration to a single mode of the radiation field, for single-photon absorption by a chiral molecule in its ground state in the presence of an LG beam, the initial and final states of the molecule-radiation system are $|i\rangle=\left|E_{0}\right\rangle|n(\boldsymbol{k}, \eta, \ell, p)\rangle$ and $|f\rangle=\left|E_{f}\right\rangle|n(k, \eta, \ell, p)\rangle$, respectively. 


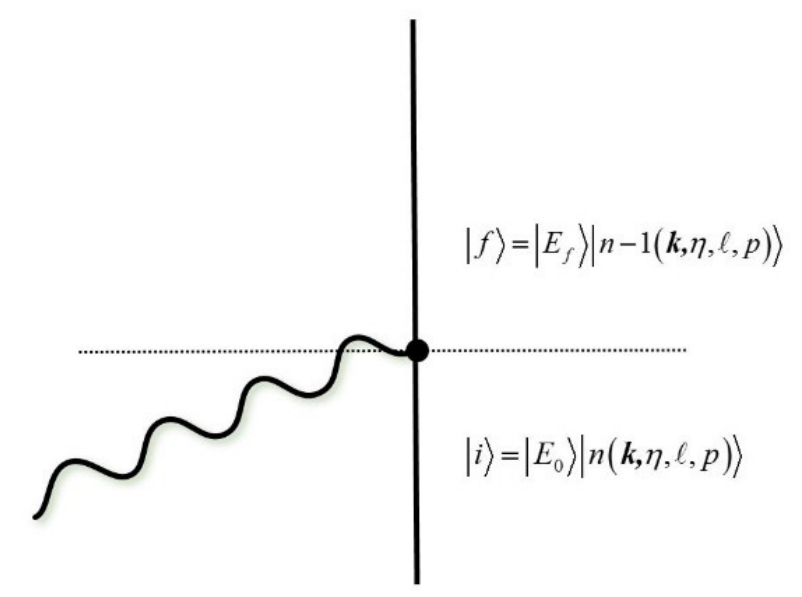

Figure 1: Time-ordered Feynman diagram for single-photon absorption by a molecule initially in its ground state in the presence of $n$ photons of a specific LG mode.

As exhibited by the time-ordered Feynman diagram in Figure 1, single-photon absorption is first-order in $H_{\text {int }}$ and therefore, using standard perturbation methods, the matrix element for process can be written as

$$
\begin{aligned}
M_{f i} & =\left\langle f\left|H_{\mathrm{int}}\right| i\right\rangle \\
& =-\varepsilon_{0}^{-1} \mu_{i}^{f 0}(\xi)\left\langle(n-1)\left|d_{i}^{\perp}(\boldsymbol{r})\right| n\right\rangle-m_{i}^{f 0}(\xi)\left\langle(n-1)\left|b_{i}(\boldsymbol{r})\right| n\right\rangle-\varepsilon_{0}^{-1} Q_{i j}^{f 0}(\xi)\left\langle(n-1)\left|\nabla_{j} d_{i}^{\perp}(\boldsymbol{r})\right| n\right\rangle,
\end{aligned}
$$

where the mode-dependence for the radiation state has been dropped for clarity. As alluded to earlier, it is the inclusion of the electric quadrupole E2 interacting with the radiation field which produces couplings that have a dependence on the sign of $\ell$. To derive this, we must first concentrate on the final term in (6), as it is necessary to carry out the gradient operation $\nabla_{j}$ on the field. It is this dependence on the gradient of the electric field which is, to this level of multipole expansion, unique to E2 moments: neither the E1 or M1 terms possess this characteristic. First, using (3), the E2 contribution can be written as

$$
-\varepsilon_{0}^{-1} Q_{i j}^{f 0}\left\langle(n-1)\left|\nabla_{j} d_{i}^{\perp}(\boldsymbol{r})\right| n\right\rangle=-i\left(\frac{n \hbar c k}{2 \varepsilon_{0} V}\right)^{1 / 2} f_{\ell, p}(r) e_{i} Q_{i j}^{f 0} \nabla_{j} \mathrm{e}^{(i k z+i \ell \phi)}
$$

The operator $\nabla_{j}$ is given in cylindrical coordinates as

$$
\nabla_{j}=\frac{\partial}{\partial r} \hat{r}_{j}+\frac{1}{r} \frac{\partial}{\partial \phi} \hat{\phi}_{j}+\frac{\partial}{\partial z} \hat{z}_{j}
$$


To exemplify the effect that the gradient operation (8) has on (7), the relevant quantities are isolated, and the operation, with the aid of the product rule and $\partial \hat{\phi} / \partial \phi=-\hat{r}$, is thus seen to be

$$
\begin{aligned}
\nabla_{j} f_{\ell, p}(r) \mathrm{e}^{(i k z+i \ell \phi)} & =f_{\ell, p}(r) \frac{\partial}{\partial r} \hat{r}_{j} \mathrm{e}^{(i k z+i \ell \phi)}+f_{\ell, p}(r) \frac{1}{r} \frac{\partial}{\partial \phi} \hat{\phi}_{j} \mathrm{e}^{(i k z+i \ell \phi)}+f_{\ell, p}(r) \frac{\partial}{\partial z} \hat{z}_{j} \mathrm{e}^{(i k z+i \ell \phi)} \\
& =\hat{r}_{j} \frac{\partial f_{\ell, p}(r)}{\partial r} \mathrm{e}^{(i k z+i \ell \phi)}+f_{\ell, p}(r) \frac{1}{r}\left(i \ell \hat{\phi}_{j}-\hat{r}_{j}\right) \mathrm{e}^{(i k z+i \ell \phi)}+f_{\ell, p}(r) i k \hat{z}_{j} \mathrm{e}^{(i k z+i \ell \phi)}
\end{aligned}
$$

Therefore, this result (9) can be inserted into (7) to give

$$
-\varepsilon_{0}^{-1} Q_{i j}^{f 0}\left\langle(n-1)\left|\nabla_{j} d_{i}^{\perp}(\boldsymbol{r})\right| n\right\rangle=-i\left(\frac{n \hbar c k}{2 \varepsilon_{0} V}\right)^{1 / 2} f_{\ell, p}(r) e_{i} Q_{i j}^{f 0}\left[\frac{1}{f_{\ell, p}(r)} \frac{\partial f_{\ell, p}(r)}{\partial r} \hat{r}_{j}+\frac{1}{r}\left(i \ell \hat{\phi}_{j}-\hat{r}_{j}\right)+i k \hat{z}_{j}\right] \mathrm{e}^{(i k z+i \ell \phi)},
$$

which upon inserting back into (6), and carrying out E1 and M1 field operations - which are not dependent upon the gradient of the electric field - gives the total quantum amplitude as

$$
M_{f i}=-i\left(\frac{n \hbar c k}{2 \varepsilon_{o} V}\right)^{1 / 2} f_{\ell, p}(r)\left[e_{i} \mu_{i}^{f 0}+\frac{1}{c} b_{i} m_{i}^{f 0}+e_{i} Q_{i j}^{f 0}\left(\hat{r}_{j} \frac{1}{f_{\ell, p}(r)} \frac{\partial f_{\ell, p}(r)}{\partial r}+\frac{1}{r}\left(i \ell \hat{\phi}_{j}-\hat{r}_{j}\right)+i k \hat{z}_{j}\right)\right] \mathrm{e}^{(i k z+i \ell \hat{\phi})} .
$$

As it stands, in (11) there is a clear dependence on the magnitude, and more importantly the sign of $\ell$ in the phase factor common to all three terms in the square bracket, and in addition there is also a linear dependence on $\ell$ present in the third term in square brackets: the quadrupole $\mathrm{E} 2$ term.

To proceed, we require the use of the Fermi rule to calculate the rate of the optical process: $\Gamma \approx\left|M_{f i}\right|^{2}$. Evidently this calculation requires the modulus square of the matrix element (11) to be taken. This being the case, the dependence on $\ell$ present within the phase factor is seen to vanish: $\left|\mathrm{e}^{i \ell \phi}\right|^{2}=\mathrm{e}^{i \ell \phi} \mathrm{e}^{-i \ell \phi}=1$. If, as in previous studies ${ }^{13}$, only the E1 and M1 terms of (11) were included, it can be seen that for the rate of single-photon absorption there would be no dependence on the handedness of the vortex beam through the sign of $\ell$. However, due to the inclusion of E2 moments, terms with a dependence on the sign of $\ell$ will survive the squaring of the quantum amplitude. In fact, taking the modulus square of (11), produces a plethora of coupling terms: namely, E1E1, M1M1, E1M1, E1E2, M1E2, and E2E2. The terms E1E1 and M1M1 are the standard contributions to single-photon absorption in the dipole approximation, neither being sensitive to any handedness present within the system. The E1M1 term is discriminatory, but only with regards to the SAM manifest through circularly polarized photons: it is the term that gives rise to standard circular dichroism (CD).

Clearly, any term that has a dependence on E2 has the capacity to be sensitive to the sign of $\ell$. This then leaves us to concentrate on the terms E1E2, M1E2, and E2E2. We are now at a suitable juncture to establish the spatial parity of these multipole moment products. At the outset, we explicitly state the use of a chiral matter, and we are interested in 
whether switching one chiral molecule with its enantiomer would produce a discriminatory effect on the optical rate of single-photon absorption. The spatial parity signatures of the multipole moments and their products are listed in Table 1.

\begin{tabular}{|c|c|}
\hline Molecular parameter & Spatial parity signature \\
\hline E1, E1M1, E1E2 & - \\
\hline $\begin{array}{c}\text { M1, E2, E1E1, M1M1, } \\
\text { M1E2, E2E2 }\end{array}$ & + \\
\hline
\end{tabular}

Table 1: Spatial parity signatures of various molecular multipole transition moments and products.

The M1E2 and E2E2 multipole products have even parity with regards to spatial inversion symmetry operation, and therefore replacing one chiral molecule with its corresponding enantiomer of opposite handedness (equivalent to spatial inversion) leaves the sign of these multipole moment products invariant. However, for the E1E2 couplings, which possess an odd parity signature for the spatial inversion symmetry operation, the switching of enantiomers will lead to a result that differs in sign, and is therefore discriminatory with regards to the material component of the system. Of course, for any specific optical phenomena, the total parity signature of radiation-matter system has to be evaluated before any physically observable chiroptical interaction can be anticipated.

In the current work, we are concerned with chiral media, and therefore we can concentrate upon the E1E2 terms, as only these multipolar products exhibit the capacity to change sign upon replacing a chiral molecule with its corresponding enantiomer. The M1E2 and E2E2 terms, do however, appear to account for a chiroptical sensitivity - but only with regards to the radiation part of the system. These terms, therefore, show a capacity to generate discriminatory effects in achiral material with regards to the relative handedness of the polarization and topological charge. ${ }^{21}$ This represents an area that clearly warrants further investigation, and we return to the issue in the discussion section of this Proceedings. It is also worth commenting on the magnitude of these couplings. In general, the M1 and E2 couplings are smaller in magnitude by a factor of the order of the fine-structure constant $\alpha \approx 1 / 137$ compared to the leading order E1 interaction. Therefore, the E1E2 is a factor of $\alpha$ larger than the M1E2 and E2E2 terms, which further validates the current emphasize on the E1E2 terms.

Continuing, we see that the two relevant E1E2 terms are

$$
\mathrm{E} 1 \overline{\mathrm{E}} 2=\left(\frac{n \hbar c k}{2 \varepsilon_{o} V}\right) f_{\ell, p}{ }^{2}(r) e_{i} \bar{e}_{k} \mu_{i}^{f 0} \bar{Q}_{k j}^{f 0}\left(\hat{r}_{j} \frac{1}{f_{\ell, p}(r)} \frac{\partial f_{\ell, p}(r)}{\partial r}+\frac{1}{r}\left(-i \ell \hat{\phi}_{j}-\hat{r}_{j}\right)-i k \hat{z}_{j}\right),
$$

and

$$
\overline{\mathrm{E}} 1 \mathrm{E} 2=\left(\frac{n \hbar c k}{2 \varepsilon_{o} V}\right) f_{\ell, p}{ }^{2}(r) e_{i} \bar{e}_{k} \bar{\mu}_{k}^{f 0} Q_{i j}^{f 0}\left(\hat{r}_{j} \frac{1}{f_{\ell, p}(r)} \frac{\partial f_{\ell, p}(r)}{\partial r}+\frac{1}{r}\left(i \ell \hat{\phi}_{j}-\hat{r}_{j}\right)+i k \hat{z}_{j}\right) .
$$


Collecting all the terms that exhibit a dependence on the sign of $\ell$ in (12) and (13), and invoking all the components of the Fermi rule leads to a modified rate

$$
\Gamma^{\prime}(\ell)=\frac{I(\omega) N}{2 c \hbar^{2} \varepsilon_{o}} \frac{1}{r} f_{\ell, p}^{2}(r) e_{i} \bar{e}_{k} i \ell \hat{\phi}_{j}\left(\mu_{k}^{f 0} Q_{i j}^{f 0}-\mu_{i}^{f 0} Q_{k j}^{f 0}\right),
$$

where $I(\omega)$ is the input beam irradiance per unit frequency interval and $N$ is the number of absorbing chiral molecules. Both $\boldsymbol{\mu}^{f 0}$ and $\boldsymbol{Q}^{f 0}$ are real quantities, and therefore the polarization vectors in (14) must be complex quantities in order to secure a non-zero real result. This leads to the remarkable conclusion that, to the leading order of E1E2, we can only anticipate a chiroptical response to the handedness of the vortex beam if that same beam is circularly polarized. This means that the OAM of light alone cannot produce chiroptical effects, and that only in conjunction with SAM is it possible to engage the vortex handedness in discriminatory interactions with chiral molecules. It is for this reason that the optical process described by (14) is an analogue of circular dichroism (CD), but with beams carrying a topological charge. From henceforth, we refer to this novel effect whereby the topological charge engages with and modifies the extent of chiroptical discrimination as circular-vortex dichroism (CVD). ${ }^{22}$

Further manipulation of (14) using the following identity

$$
e_{i}^{\mathrm{L} / \mathrm{R}} \bar{e}_{k}^{\mathrm{L} / \mathrm{R}}=\frac{1}{2}\left[\left(\delta_{i k}-\hat{k}_{i} \hat{k}_{k}\right) \mp i \varepsilon_{i k m} \hat{k}_{m}\right],
$$

allows the rate to be secured in a form where the influence of circular polarization handedness is readily identifiable:

$$
\Gamma^{\prime(\mathrm{L} / \mathrm{R})}(\ell)= \pm \frac{I(\omega) N}{4 c \hbar^{2} \varepsilon_{o}} \frac{1}{r} f_{\ell, p}^{2}(r) \varepsilon_{i k m} \hat{k}_{m} \ell \hat{\phi}_{j}\left(\mu_{k}^{f 0} Q_{i j}^{f 0}-\mu_{i}^{f 0} Q_{k j}^{f 0}\right)
$$

where the \pm designates either left-handed or right-handed polarization, respectively. To enable us to elucidate the physicality of the CVD effect, it is more suitable to express a rate which incorporates the CVD terms, as well as the standard CD process. This is a legitimate step as the detailed analysis reveals that structured beams still produce the standard CD response, in addition to the CVD response. To proceed we cast the result in terms of the absolute difference in absorption rates between left- and right-handed circularly polarized photons:

$$
\Gamma^{(\mathrm{L})}-\Gamma^{(\mathrm{R})}=\Delta \Gamma_{\mathrm{CD}}+\frac{I(\omega) N}{2 c \hbar^{2} \varepsilon_{o}} \frac{1}{r} f_{\ell, p}{ }^{2}(r) \varepsilon_{i k m} \hat{k}_{m} \ell \hat{\phi}_{j}\left(\mu_{i}^{f 0} Q_{k j}^{f 0}+\mu_{k}^{f 0} Q_{i j}^{f 0}\right),
$$

where $\Delta \Gamma_{\mathrm{CD}}$ includes both the E1E2 and E1M1 contributions to standard CD: 


$$
\Delta \Gamma_{\mathrm{CD}}=\frac{2 \pi N}{\hbar}\left(\frac{n \hbar c k}{2 \varepsilon_{0} V}\right) \rho_{f} f_{\ell, p}{ }^{2}(r)\left[\frac{1}{c}\left(\delta_{i k}-\hat{k}_{i} \hat{k}_{k}\right)\left(\mu_{i}^{f 0} \bar{m}_{k}^{\prime f 0}-\bar{\mu}_{k}^{f 0} m_{i}^{\prime f 0}\right)-\varepsilon_{i k m} \hat{k}_{m} k \hat{z}_{j}\left(\mu_{i}^{f 0} Q_{k j}^{f 0}-\mu_{k}^{f 0} Q_{i j}^{f 0}\right)\right]
$$

where $m_{i}^{\prime f 0}=i m_{i}^{f 0}$. Another reason it is important to include the CD terms in the overall rate is that is the trivial demonstration that when the beam possesses no OAM, the terms dependent on $\ell$ clearly vanish, leaving terms which account for $\mathrm{CD}$ in precise agreement with the standard result (though of course differing slightly in form due to the accounting for the beam structure) ${ }^{23,7}$ The magnitude of the CVD and CD will be of a similar order of magnitude since the E2 and M1 interactions with the field are approximately equal in strength. However, (17) highlights the uniqueness of how the CVD contribution scales linearly with $\ell$. This increasing significance of the E2 interactions as the topological charge increases can be expected due to the fact that quadrupole transition moments engage with the gradient of the electric field. This enhanced role of E2 interactions with vortex beams has been recognized previously in studies involving atoms. ${ }^{24,25}$

The result (17) is applicable to one or molecules that possess a fixed orientation at any point within the LG beam. The $\hat{\phi}_{j}$ term contracts with an index of the quadrupole transition moment (which itself has a fixed orientation within the molecule), and therefore the magnitude and sign of the CVD differential will in general vary about the beam axis. More specifically, the CVD will vary between a maximum and minimum of opposite sign across the beam profile. The CVD effect therefore registers the varying directions of phase gradient around each intensity ring for an LG beam, as experienced by chiral molecules possessing a common orientation - see Figure 2.

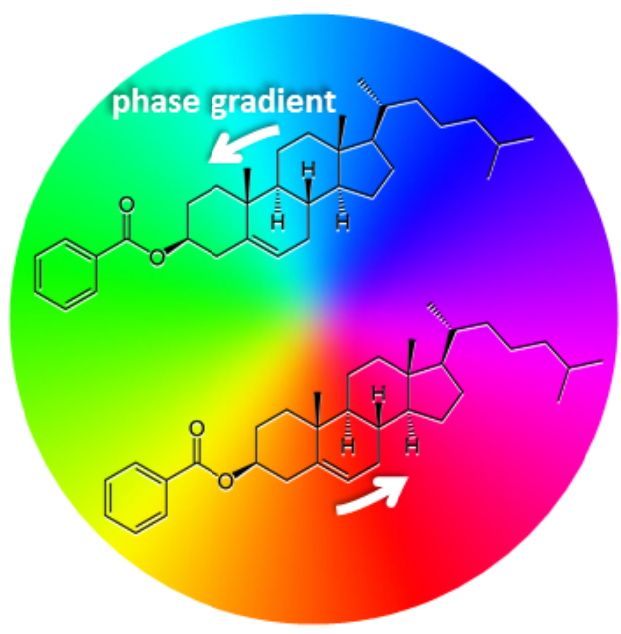

Figure 2: Illustration of the different directional sense of the phase gradient experienced by locally ordered chiral molecules (here represented by cholesteryl benzoate, an archetype molecule possessing the readiness to form liquid crystals) sited at different locations in a cross-section around the axis of a vortex beam - color here represents the optical phase of a beam with topological charge $\ell=1$.

If a system a molecules that possess to some extent a degree of orientational order, such as a poled liquid crystal or the boundaries of an isotropic medium, then the absorption differential will be enhanced on one side of the beam and equally diminished on the other - as compared with the standard rate of $\mathrm{CD}$. However, it is worth bearing in mind that due to the radial and azimuthal components being symmetrical about the $z$ axis, any integration over the whole beam profile will leave terms that are solely dependent on $\hat{z}$ (out of those dependent linearly on the unit vectors): namely, the standard CD 
terms. Thus, when looking at the whole beam profile, the CVD has a vanishing net result, and it is to be realized that the effect itself may be deemed local, being exhibited only when the extent of absorption is resolved at different locations within the beam profile.

To complete the analysis, we now entertain the possibility that the molecules are freely tumbling and the system has a lack of any orientational order. This is an almost ubiquitous state for molecular fluids, with the constituent molecules being completely randomly oriented. To secure this averaged result, we have to perform an isotropic rotational average

of the expression (16). Utilising standard techniques ${ }^{26}$, the calculation required entails a contraction of the molecular multipole E1E2 product terms with the corresponding third-rank isotropic tensor, namely the Levi-Civita epsilon:

$$
\left\langle\Gamma^{(\mathrm{L} / \mathrm{R})}(\ell)\right\rangle \approx\left\langle\mu_{k}^{f 0} Q_{i j}^{f 0}-\mu_{i}^{f 0} Q_{k j}^{f 0}\right\rangle \approx \varepsilon_{i j k} \varepsilon_{\lambda \mu v}\left(\mu_{v}^{f 0} Q_{\lambda \mu}^{f 0}-\mu_{\lambda}^{f 0} Q_{v \mu}^{f 0}\right)
$$

However, since the electric quadrupole moment is symmetric in its indices, and the Levi-Civita fully index antisymmetric, the molecular average is zero. It can therefore be concluded that the CVD is non-zero for molecular systems possessing a degree of orientational order, but vanishes for randomly orientated molecules.

\section{DISCUSSION}

One of the more striking results from the analysis is the requirement of circularly polarized light to enable the vortex handedness to play a role in discriminatory single-photon absorption. This interplay between SAM and OAM is an interesting facet of the work, and gives rise to questions about its physical interpretation. The issue may be explained through a spin-orbit interaction approach ${ }^{27-29}$, and could potentially lead to further interesting applications. ${ }^{30}$ Another issue which requires future work involves looking at the E2E2 and M1E2 terms which appear to suggest the possibility of a chiroptical response even with achiral media ${ }^{21,15}$ : this is a prospect that chiral sensitivity might arise solely from the total angular momentum possessed by the radiation components of the system, exhibiting. an interplay of the polarization state and topological charge of the vortex beam. Such an effect can be expected to persist even in systems comprising randomly oriented molecules.

The aim of this paper has been to establish a broader basis for the analysis of the role twisted beams can play in chiroptical interactions. It has been explicitly shown that - to the most significant order of multipole moment - the electric quadrupole E2 moment must be engaged in order to potentially exhibit discriminatory behaviour with regards to the sign of wavefront handedness of a vortex beam. The results specifically show that the sign and magnitude of $\ell$ plays a significant role in the local rate of one-photon absorption in oriented chiral systems, an effect termed circular-vortex dichroism (CVD). The influence vanishes for media that is completely randomly oriented. The inclusion of electric quadrupole transition moments in conjunction with beams of structured light may now be anticipated to lead to other discriminatory effects which possess a sensitivity to the topological charge. Indeed, recent experiments suggest a role in circular differential Raman scattering ${ }^{31}$ and the extension of the principles outlined in this work to further and more complex optical processes in both chiral and achiral media constitute the direction for further study. 


\section{REFERENCES}

[1] Andrews, D. L. and Babiker, M., [The Angular Momentum of Light], Cambridge University Press, Cambridge, UK (2013).

[2] Rubinsztein-Dunlop, H., Forbes, A., Berry, M., Dennis, M., Andrews, D. L., Mansuripur, M., Denz, C., Alpmann, C., Banzer, P. and Bauer, T., “Roadmap on structured light,” J. Opt. 19, 013001 (2016).

[3] Barnett, S. M., Babiker, M. and Padgett, M. J., [Optical orbital angular momentum] The Royal Society, (2017).

[4] Darwin, C., "Notes on the theory of radiation," Proc. R. Soc. A 136, 36-52 (1932).

[5] Allen, L., Beijersbergen, M. W., Spreeuw, R. J. C. and Woerdman, J. P., "Orbital angular momentum of light and the transformation of Laguerre-Gaussian laser modes," Phys. Rev. A 45, 8185-8189 (1992).

[6] Coles, M. M., "An Upper Bound on the Rate of Information Transfer in Optical Vortex Beams," arXiv preprint arXiv:1703.08718, (2017).

[7] Craig, D. P. and Thirunamachandran, T., [Molecular Quantum Electrodynamics: An Introduction to RadiationMolecule Interactions], Dover Publications, Mineola, NY (1998).

[8] Berova, N., Nakanishi, K. and Woody, R., [Circular dichroism: principles and applications], John Wiley \& Sons, (2000).

[9] Yokoyama, A., Yoshida, M., Ishii, A. and Kato, Y., "Giant circular dichroism in individual carbon nanotubes induced by extrinsic chirality,” Phys. Rev. X 4, 011005 (2014).

[10] Barron, L. D., [Molecular Light Scattering and Optical Activity], Cambridge University Press Cambridge, UK (2004).

[11] Bradshaw, D. S., Forbes, K. A., Leeder, J. M. and Andrews, D. L., "Chirality in optical trapping and optical binding," Photonics 2, 483-497 (2015).

[12] Forbes, K. A. and Andrews, D. L., "Chiral discrimination in optical binding," Phys. Rev. A 91, 053824 (2015).

[13] Andrews, D. L., Dávila Romero, L. C. and Babiker, M., "On optical vortex interactions with chiral matter," Opt. Commun. 237, 133-139 (2004).

[14] Andrews, D. L. and Babiker, M., "Quantum electrodynamics, angular momentum and chirality," in [The Angular Momentum of Light], D. L. Andrews and M. Babiker, eds. (Cambridge University Press, New York, 2012), pp. 246-263.

[15] Afanasev, A., Carlson, C. E. and Solyanik, M., "Circular Dichroism of Twisted Photons in the Non-Chiral Atomic Matter," J. Opt. 19, 105401 (2017).

[16] Brullot, W., Vanbel, M. K., Swusten, T. and Verbiest, T., "Resolving enantiomers using the optical angular momentum of twisted light," Science Advances 2, e1501349 (2016).

[17] Araoka, F., Verbiest, T., Clays, K. and Persoons, A., "Interactions of twisted light with chiral molecules: an experimental investigation," Phys. Rev. A 71, 055401 (2005).

[18] Löffler, W., Broer, D. and Woerdman, J., "Circular dichroism of cholesteric polymers and the orbital angular momentum of light," Phys. Rev. A 83, 065801 (2011).

[19] Forbes, K. A., Bradshaw, D. S. and Andrews, D. L., "Identifying diamagnetic interactions in scattering and nonlinear optics," Phys. Rev. A 94, 033837 (2016).

[20] Dávila Romero, L. C., Andrews, D. L. and Babiker, M., "A quantum electrodynamics framework for the nonlinear optics of twisted beams," J. Opt. B: Quantum Semiclass. Opt. 4, S66-S72 (2002).

[21] Zambrana-Puyalto, X., Vidal, X. and Molina-Terriza, G., "Angular momentum-induced circular dichroism in non-chiral nanostructures," Nat. Commun. 5, 4922 (2014).

[22] Forbes, K. A. and Andrews, D. L., “Optical orbital angular momentum: twisted light and chirality,” Opt. Lett. 43, 435-438 (2018).

[23] Power, E. A. and Thirunamachandran, T., "Circular dichroism: A general theory based on quantum electrodynamics," J. Chem. Phys. 60, 3695-3701 (1974).

[24] Lembessis, V. and Babiker, M., "Enhanced quadrupole effects for atoms in optical vortices," Phys. Rev. Lett. 110, 083002 (2013).

[25] Afanasev, A., Carlson, C. E. and Mukherjee, A., "High-multipole excitations of hydrogen-like atoms by twisted photons near a phase singularity," J. Opt 18, 074013 (2016).

[26] Andrews, D. L. and Thirunamachandran, T., "On three-dimensional rotational averages," J. Chem. Phys. 67, 5026-5033 (1977). 
[27] Bliokh, K. Y., Rodríguez-Fortuño, F., Nori, F. and Zayats, A. V., "Spin-orbit interactions of light,” Nat. Photonics 9, 796-808 (2015).

[28] Quinteiro, G. F., Schmidt-Kaler, F. and Schmiegelow, C. T., "Twisted-Light-Ion Interaction: The Role of Longitudinal Fields," Phys. Rev. Lett. 119, 253203 (2017).

[29] Samlan, C., Suna, R. R., Naik, D. N. and Viswanathan, N. K., "Spin-orbit beams for optical chirality measurement," Appl. Phys. Lett. 112, 031101 (2018).

[30] Bhatti, D., von Zanthier, J. and Agarwal, G. S., "Entanglement of polarization and orbital angular momentum," Phys. Rev. A 91, 062303 (2015).

[31] Bendau, E., Zhang, L., Gozali, R., Ashrafi, S. and Alfano, R. R., "Vortex beams and optical activity of sucrose." Proc. SPIE 10120, 5. 